\title{
Some applications of Shisha-Mond theorem
}

\section{DAN BĂRBOSU}

\section{ABSTRACT.}

A result due to Shisha, O. and Mond, B., is recalled and some applications in the evaluation of approximation order by linear positive operator are presented.

\section{REFERENCES}

[1] Agratini, O., Approximare prin operatori liniari, Presa Univ. Clujeană, Cluj-Napoca, 2001 (Romanian)

[2] Anastassiou, G., A., Generalized Shisha-Mond type inequalities, An. St. Univ. Oradea, Fasc. Matematica, Tome XV (2008), 141-143

[3] Anastassiou, G., A., A generalization of Shisha-Mond type inequality, Intelligent Mathematics: Computational Analysis, Intelligent Systems Reference Library, Vol 5 (2011), 273-274

[4] Bărbosu, D., Aproximarea funcţiilor de mai multe variabile prin sume booleene de operatori liniari de tip interpolator, Ed. Risoprint, Cluj-Napoca, 2002 (Romanian)

[5] Bernstein, S. N., Demonstration du théoreme de Weierstrass fondée sur le calcul des probabilités, Commun. Soc. Math. Kharkow, 13 (1912-1913), No. $2,1-2$

[6] Favard, I., Sur le multiplicateurs d' interpolation, J. Math. Pure Appl., 23 (1944), No. 9, 219-247

[7] Mirakjan, G. M., Approximation of continuous functions with the aid of polynomials (Russian), Dokl. Akad. Nauk. SSSR, 31 (1941), 201-205

[8] Mortici, C., An extension of the Szász-Mirakjan operators, An. Ştiinţ. Univ. "Ovidius" Constanţa Ser. Mat., 17 (2009), No. 1, 137-143

[9] Muraru, C. V. and Acu, A. M., Some approximation properties of q-Durrmeyer-Schurer operators, Sci. Stud. Res. Ser. Math. Inform., 23 (2013), No. $1,77-84$

[10] Pop, O. T., Miclăuş, D. and Bărbosu, D., The Voronovskaja type theorem for a general class of Szász-Mirakjan operators, Miskolc Math. Notes, 14 (2013), No. 1, 219-231

[11] Popoviciu, T., Sur l'approximation des fonctions convexes d'ordre supérieur Mathematica (Cluj), 10 (1935), 49-54

[12] Shisha, O. and Mond, B., The degree of approximation to periodic functions by linear positive operators, Proc. Nat. Acad. Sci. U.S.A., 60 (1968), $1196-1200$

[13] Stancu, D. D., Asupra unei generalizări a polinoamelor lui Bernstein, Studia Univ. Babeş-Bolyai, 14 (1969), No. 2, 31-45 (Romanian)

[14] Stancu, F., Aproximarea funcţiilor de doua şi mai multe variabile cu ajutorul operatorilor liniari şi pozitivi, Ph D. Thesis, Cluj-Napoca, 1984 (Romanian)

[15] Stancu, D. D., Coman, Gh. and Agratini, O., Trîmbiţaş, R., Analiză numerică şi teoria aproximării I, Presa Univ. Clujeană, Cluj-Napoca, (2002) (Romanian)

[16] Szász, O., Generalization of S. Bernstein's polynomials to the infinite interval, J. Researck, National Burean of Standards, 45 (1950), $239-245$

[17] Swetits, J. J. and Wood, B., Unbounded functions and positive linear operators, J. Approx. Theory, Vol. 34 (1982), Issue 4, 325-334

Department of Mathematics And Computer SCience

FACUlty of SCIENCES NORTH University CENTER AT Baia MARE

TECHNICAL UNIVERSITY OF CLUJ-NAPOCA

VICTORIEI 76, 430122 BAIA MARE, ROMANIA

E-mail address: barbosudan@yahoo.com 